\title{
THE FEFFERMAN METRIC AND PSEUDOHERMITIAN INVARIANTS
}

\author{
JOHN M. LEE ${ }^{1}$
}

\begin{abstract}
C. Fefferman has shown that a real strictly pseudoconvex hypersurface in complex $n$-space carries a natural conformal Lorentz metric on a circle bundle over the manifold. This paper presents two intrinsic constructions of the metric, valid on an abstract CR manifold. One is in terms of tautologous differential forms on a natural circle bundle; the other is in terms of Webster's pseudohermitian invariants. These results are applied to compute the connection and curvature forms of the Fefferman metric explicitly.
\end{abstract}

1. Introduction. In $1976 \mathrm{C}$. Fefferman [5] showed that, if $M$ is a real strictly pseudoconvex hypersurface in $\mathbf{C}^{n}, M \times S^{1}$ carries a Lorentz metric whose conformal class is invariant under biholomorphisms. The null geodesics ("light rays") of this metric project to the biholomorphically invariant curves on $M$ called chains. It is therefore of considerable interest to find ways of characterizing the Fefferman metric in terms of the intrinsic CR structure of $M$, thereby extending its definition to abstract CR manifolds.

The first such characterization was obtained by D. Burns, K. Diederich, and S. Shnider [2], who showed how to obtain the Fefferman metric from the Chern connection in the Chern "CR structure bundle" of $M$. Their construction is difficult to work with, however, since the metric appears first as a tensor in the very large CR structure bundle $Y$, and one then shows that it descends to a circle bundle $C$ obtained as a quotient of $Y$.

Recently, F. Farris [4] has given another construction of the Fefferman metric, working only within the circle bundle $C$. His construction, however, required the assumption of the existence of a closed $(n+1,0)$-form on $M$. It is easy to find such a form when $M$ is embedded in $\mathbf{C}^{n+1}$, but $\mathrm{H}$. Jacobowitz has shown [7a] that there may not exist one on a general CR manifold. If there is no such form, Farris' construction only gives the Fefferman metric at a point in terms of the coefficients in a formal power series approximation to a closed $(n+1,0)$-form.

This paper presents two new characterizations of the Fefferman metric, valid on an abstract CR manifold, which avoid these difficulties. Both characterizations begin with a choice of a one-form annihilating the maximal complex subspace of $M$ (a "pseudohermitian structure"), and define Lorentz metrics on an intrinsically defined circle bundle $C$ over $M$.

The first characterization is in terms of differential forms which are intrinsic to the circle bundle $C$ and defined by simple normalizations relating them to the chosen pseudohermitian structure. The second is in terms of the Webster connection forms

Received by the editors September 4, 1985.

1980 Mathematics Subject Classification. Primary 32F25.

${ }^{1}$ Research suported in part by an NSF postdoctoral research fellowship. 
of the pseudohermitian structure. It is then shown that these two Lorentz metrics are the same, that they transform conformally under a change of pseudohermitian structure, and that they agree with the Fefferman metric in case $M$ is embedded in $\mathbf{C}^{n+1}$.

The first of these two characterizations shows clearly the relation of the intrinsically defined metric to Fefferman's original extrinsic construction. In particular, the two normalizations which characterize the metric on $C$ correspond directly to Fefferman's first and second formal power series solutions to the complex MongeAmpère equation. The second characterization makes the metric easily accessible to intrinsic computations, using the formalism of Webster's pseudohermitian connection and covariant derivatives.

In $\S 2$ we recall basic facts and notations concerning CR manifolds. $\S 3$ presents the first characterization of the metric. $\S 4$ is a review of the Webster-Stanton connection of a pseudohermitian structure, which is used in $\S 5$ to deduce the second characterization. Finally, in $\S 6$ we apply these results to compute curvature invariants of the Fefferman metric in terms of Webster curvature invariants of the corresponding pseudohermitian structure. In particular, it is shown that the scalar curvature of the Fefferman metric is a constant multiple of the Webster scalar curvature, and we state without proof a result showing the relationship of the Ricci curvature of the Fefferman metric to Webster's Ricci and torsion tensors.

These characterizations of the metric and its curvature invariants will be of value in further studies of the behavior of chains, since they allow the geodesic equations to be written much more explicitly than has heretofore been possible. In addition, R. Graham [6a] has recently applied these results to obtain a new proof of Sparling's characterization of Lorentz metrics which arise as Fefferman metrics of CR manifolds.

The simple relationships between the curvature of the Fefferman metric and that of the Webster-Stanton connection suggest several interesting geometric problems for CR manifolds. For example, the fact that the Webster scalar curvature and Fefferman scalar curvature agree up to a constant multiple means that the Yamabe problem for the Fefferman metric on $C$ (i.e. to find a conformal representative of the Fefferman metric which has constant scalar curvature) can be reduced to a problem on the CR manifold $M$. While the former problem is decidedly nonelliptic, the latter is at least subelliptic. This problem is the subject of a pair of joint papers with D. Jerison $[\mathbf{8}, \mathbf{9}]$.

Similarly, one can ask whether one can choose the pseudohermitian structure so that the Ricci tensor of the Fefferman metric assumes a particularly simple form. The formula for Ricci tensor given in $\S 6$ shows that the Fefferman metric is never Einstein; it is not clear at this point what is the natural normalization for the Ricci tensor. On the other hand, one can ask whether a suitable choice of pseudohermitian structure will make the Webster-Ricci tensor a scalar multiple of the Levi form; this question will be dealt with in a forthcoming paper.

I am indebted to Richard Melrose, who first suggested the approach to the Fefferman metric which evolved into the first characterization given here; to Frank Farris, upon whose results the present work builds; to Robin Graham, who showed me how to simplify the proof of Theorem 6.2; and especially to David Jerison, 
whose suggestion to look for a CR-invariant Laplacian led to the key insight on which this work is based (see $[\mathbf{8}, \mathbf{9}]$ for more on these matters).

2. Pseudohermitian structures. In this section we collect some facts and notations concerning pseudohermitian structures on CR manifolds.

Let $M$ be a real, $(2 n+1)$-dimensional, orientable, $C^{\infty}$ manifold. A CR structure on $M$ is an $n$-dimensional complex subbundle $T_{1,0}$ of the complexified tangent bundle CTM satisfying $T_{1,0} \cap T_{0,1}=\{0\}$, where $T_{0,1}=\bar{T}_{1,0}$. We set

$$
H=\operatorname{Re}\left(T_{1,0} \oplus T_{0,1}\right),
$$

so that $H$ is a $2 n$ (real) dimensional subbundle of $T M$. $H$ carries a natural complex structure map $J: H \rightarrow H$ given by $J(V+\bar{V})=i(V-\bar{V})$ for $V \in T_{1,0}$. We will assume throughout that the CR structure is integrable, that is, $T_{1,0}$ satisfies the formal Frobenius condition $\left[T_{1,0}, T_{1,0}\right] \subset T_{1,0}$.

The most important example of an integrable CR structure is of course that induced by an embedding $M \subset \mathbf{C}^{n+1}$, in which case $T_{1,0}=T_{1,0} \mathbf{C}^{n+1} \cap \mathbf{C T M}$.

The bundle $\Omega^{q, 0}$ of complex $(q, 0)$-forms on $M$ is defined by

$$
\left.\Omega^{q, 0}=\left\{\eta \in \mathbf{C} \wedge^{q} M: V\right\rfloor \eta=0 \text { for } V \in T_{0,1}\right\} .
$$

Of particular interest is $\Omega^{n+1,0}$, which has complex fiber dimension one. We set $K=\Omega^{n+1,0}$, and call it the canonical bundle of $M$. If $M$ obtains its CR structure from an embedding in a complex manifold $\Omega, K$ is naturally isomorphic to the restriction of the canonical bundle $K_{\Omega}$ of $(n+1,0)$-forms on $\Omega$.

Let $E \subset T^{*} M$ denote the real line bundle $H^{\perp}$. Because we assume $M$ is orientable, and the complex structure $J$ induces an orientation on $H, E$ has a global nonvanishing section. A choice of such a 1 -form $\theta$ is called a pseudohermitian structure on $M$. Associated with $\theta$ is the Hermitian form $L_{\theta}$ on $T_{1,0}$ :

$$
L_{\theta}(V, \bar{W})=-i d \theta(V \wedge \bar{W}),
$$

called the Levi form of $\theta$. This can also be written

$$
L_{\theta}(V, \bar{W})=d \theta(V \wedge J \bar{W}) ;
$$

in this form $L_{\theta}$ extends by complex linearity to a symmetric form on $\mathbf{C H}$, real on $H$, which we denote also by $L_{\theta}$.

Of course, the Levi form depends on the choice of $\theta$, but it is CR-invariant up to a conformal multiple. If $L_{\theta}$ is definite, $M$ is said to be strictly pseudoconvex. In this case, it is natural to orient $E$ by declaring a section $\theta$ to be positive if $L_{\theta}$ is positive. We will assume henceforth that $M$ is strictly pseudoconvx and $\theta$ is positive.

On a pseudohermitian manifold $M$ there is a unique vector field $T=T_{\theta}$ transverse to $H$, defined by

$$
T\rfloor d \theta=0, \quad T\rfloor \theta=1 .
$$

This defines $T$ uniquely because $d \theta$ is nondegenerate on $H$ and thus has precisely one null direction transverse to $H$.

Calculations on a pseudohermitian manifold are simplified if we work with special coframes. If $\left\{\theta^{1}, \ldots, \theta^{n}\right\}$ are $(1,0)$ forms whose restrictions to $T_{1,0}$ form a basis for $T_{1,0}^{*}$, and such that $\left.T\right\rfloor \theta^{\alpha}=0$ for $\alpha=1, \ldots, n$, we call $\left\{\theta^{\alpha}\right\}$ an admissible coframe. 
Then $\left\{\theta, \theta^{1}, \ldots, \theta^{n}, \theta^{\overline{1}}, \ldots, \theta^{\bar{n}}\right\}$, form a coframe for $\mathbf{C} T M$. With respect to an admissible coframe, the integrability condition and (2.1) imply that

$$
d \theta=i h_{\alpha \bar{\beta}} \theta^{\alpha} \wedge \theta^{\bar{\beta}}
$$

for some hermitian matrix of functions $\left(h_{\alpha \bar{\beta}}\right)$, which is positive definite if $M$ is strictly pseudoconvex. If $\left\{Z_{1}, \ldots, Z_{n}\right\}$ is the frame for $T_{1,0}$ dual to $\left\{\theta^{\alpha}\right\}$, and $V=V^{\alpha} Z_{\alpha}, W=W^{\alpha} Z_{\alpha}$ are sections of $T_{1,0}$, then the Levi form is given by $L_{\theta}(V, \bar{W})=\frac{1}{2} h_{\alpha \bar{\beta}} V^{\alpha} W^{\bar{\beta}}$.

There is a natural trace operation on 2 -forms on $M$. If $\omega$ is any 2 -form, there is a unique complex-linear map $\tilde{\omega}: T_{1,0} \rightarrow T_{1,0}$ such that for all $X, Y \in T_{1,0}$,

$$
\omega(X \wedge \bar{Y})=d \theta(\tilde{\omega} X \wedge \bar{Y}) .
$$

We define $\operatorname{Tr} \omega=\operatorname{Tr} \tilde{\omega}$. If $\left\{\theta^{\alpha}\right\}$ is an admissible coframe and $\omega=i \omega_{\alpha \bar{\beta}} \theta^{\alpha} \wedge \theta^{\bar{\beta}}$ $\left(\bmod \theta^{\alpha} \wedge \theta^{\gamma}, \theta^{\bar{\beta}} \wedge \theta^{\bar{\alpha}}, \theta\right)$, one checks easily that $\operatorname{Tr} \omega=h^{\alpha \bar{\beta}} \omega_{\alpha \bar{\beta}}$. This agrees with the definition given by Chern in [3] except for a factor of $i$; we have chosen this normalization so that $\operatorname{Tr} \omega$ is real when $\omega$ is.

$M$ carries a natural volume form $\psi=\theta \wedge d \theta^{n}$, nonvanishing because $M$ is strictly pseudoconvex, which induces an $L^{2}$ inner product on functions:

$$
\langle u, \bar{v}\rangle_{\theta}=\int_{M} u \bar{v} \psi
$$

The Levi form induces a dual metric on $H^{*}$, which we denote by $L_{\theta}^{*}$, and a norm $|\omega|_{\theta}^{2}=L_{\theta}^{*}(\omega, \omega)$. This in turn induces an $L^{2}$ inner product

$$
\langle\omega, \bar{\eta}\rangle_{\theta}=\int_{M} L_{\theta}^{*}(\omega, \bar{\eta}) \psi
$$

on sections of $H^{*}$. If $r: T^{*} M \rightarrow H^{*}$ is the natural restriction map, and $u \in C^{\infty}(M)$, we can define a secton $d_{b} u$ of $H^{*}$ by $d_{b} u=r \circ d u$.

We then define the real sublaplacian operator $\Delta_{b}$ on functions by

$$
\left\langle\Delta_{b} u, \bar{v}\right\rangle_{\theta}=\frac{1}{2}\left\langle d_{b} u, d_{b} \bar{v}\right\rangle_{\theta}, \quad v \in C_{c}^{\infty}(M) .
$$

(A. Greenleaf, in [7], defined an operator $\tilde{\Delta}$ which is the negative of $\Delta_{b}$.) Similarly, if we let $\bar{\partial}_{b} u$ denote the projection of $d_{b} u$ onto $T_{0,1}^{*}$, the complex Kohn-Spencer Laplacian $\square_{b}$ is defined as in $[\mathbf{1 0}]$ by

$$
\left\langle\square_{b} u, \bar{v}\right\rangle_{\theta}=\left\langle\bar{\partial}_{b} u, \overline{\bar{\partial}}_{b} v\right\rangle_{\theta}, \quad v \in C_{c}^{\infty}(M)
$$

The sublaplacian $\Delta_{b}$ generalizes the operator $\mathcal{L}_{0}$ defined on the Heisenberg group by Folland and Stein $[6]$. They show that in that case $\square_{b}=\mathcal{L}_{0}+i n T$ on functions. The analogous relation holds in general:

(2.3) THEOREM. $\square_{b}=\Delta_{b}+i n T$ on functions.

PROOF. Choose an admissible coframe $\left\{\theta^{\alpha}\right\}$ such that $h_{\alpha \bar{\beta}}=\delta_{\alpha \bar{\beta}}$. If $\omega=\omega_{\alpha} \theta^{\alpha}$ and $\eta=\eta_{\alpha} \theta^{\alpha}$ are sections of $T_{1,0}^{*}$, we can consider them as $(1,0)$ forms satisfying $T\rfloor \omega=T\rfloor \eta=0$. Then $L_{\theta}^{*}(\omega, \bar{\eta})=2 \omega_{\alpha} \bar{\eta}_{\alpha}$, and it follows by an easy algebraic manipulation that

$$
L_{\theta}^{*}(\omega, \bar{\eta}) \psi=2 i n \theta \wedge \omega \wedge \bar{\eta} \wedge d \theta^{n-1}
$$


Thus if $u \in C^{\infty}(M), v \in C_{c}^{\infty}(M)$ (considering $\partial_{b} u$ and $\partial_{b} v$ similarly as 1-forms),

$$
\begin{aligned}
\left\langle\Delta_{b} u, \bar{v}\right\rangle_{\theta} & =\frac{1}{2}\left\langle d_{b} u, d_{b} \bar{v}\right\rangle_{\theta} \\
& =\frac{1}{2} \int_{M} L_{\theta}^{*}\left(\partial_{b} u+\bar{\partial}_{b} u, \partial_{b} \bar{v}+\bar{\partial}_{b} \bar{v}\right) \psi \\
& =i n \int_{M}^{\theta} \theta \wedge \partial_{b} u \wedge \bar{\partial}_{b} \bar{v} \wedge d \theta^{n-1}+i n \int_{M} \theta \wedge \partial_{b} \bar{v} \wedge \bar{\partial}_{b} u \wedge d \theta^{n-1}
\end{aligned}
$$

Now by Stokes' theorem,

$$
\begin{aligned}
0 & =\int_{M} d\left(\bar{v} \theta \wedge \partial_{b} u \wedge d \theta^{n-1}\right) \\
& =\int_{M} \theta \wedge \partial_{b} u \wedge d \bar{v} \wedge d \theta^{n-1}+\int_{M} \bar{v} \partial_{b} u \wedge d \theta^{n}-\int_{M} \bar{v} \theta \wedge d \partial_{b} u \wedge d \theta^{n-1}
\end{aligned}
$$

But the first term above is equal to $\int_{M} \theta \wedge \partial_{b} u \wedge \bar{\partial}_{b} \bar{v} \wedge d \theta^{n-1}$ by type considerations, and the integrand in the second term is identically zero since it annihilates $T$. Thus

$$
\int_{M} \theta \wedge \partial_{b} u \wedge \bar{\partial}_{b} \bar{v} \wedge d \theta^{n-1}=\int_{M} \bar{v} \theta \wedge d \partial_{b} u \wedge d \theta^{n-1}
$$

On the other hand, differentiating $d u=\partial_{b} u+\bar{\partial}_{b} u+T u \theta$, we obtain

$$
d \partial_{b} u=-d\left(\bar{\partial}_{b} u+T u \theta\right)
$$

and so, applying Stokes' theorem once more,

$$
\begin{aligned}
\int_{M} \theta & \wedge \partial_{b} u \wedge \bar{\partial}_{b} \bar{v} \wedge d \theta^{n-1}=-\int_{M} \bar{v} \theta \wedge d\left(\bar{\partial}_{b} u+T u \theta\right) \wedge d \theta^{n-1} \\
& =\int_{M} \theta \wedge d \bar{v} \wedge\left(\bar{\partial}_{b} u+T u \theta\right) \wedge d \theta^{n-1}-\int_{M} \bar{v}\left(\bar{\partial}_{b} u+T u \theta\right) \wedge d \theta^{n} \\
& =\int_{M} \theta \wedge \partial_{b} \bar{v} \wedge \bar{\partial}_{b} u \wedge d \theta^{n-1}-\int_{M} \bar{v} T u \theta \wedge d \theta^{n}
\end{aligned}
$$

Inserting this into (2.4),

$$
\begin{aligned}
\left\langle\Delta_{b} u, \bar{v}\right\rangle_{\theta} & =2 i n \int_{M} \theta \wedge \partial_{b} \bar{v} \wedge \bar{\partial}_{b} u \wedge d \theta^{n-1}-i n \int_{M} \bar{v} T u \theta \wedge d \theta^{n} \\
& =\int_{M} L_{\theta}^{*}\left(\bar{\partial}_{b} u, \overline{\bar{\partial}_{b} v}\right) \psi-i n \int_{M} \bar{v} T u \psi \\
& =\left\langle\square_{b} u-i n T u, \bar{v}\right\rangle_{\theta} .
\end{aligned}
$$

Since this holds for all $v \in C_{c}^{\infty}(M)$, the theorem is proved.

3. The Fefferman metric: first characterization. We are now prepared to give a direct construction of the Fefferman metric induced by a pseudohermitian structure.

Let $(M, \theta)$ be a $(2 n+1)$-dimensional, strictly pseudoconvex pseudohermitian manifold, and $K^{*}$ the canonical bundle of $M$ with the zero section deleted. We define an intrinsic circle bundle $C=K^{*} / \mathbf{R}^{+}$as the quotient of $K^{*}$ by the natural $\mathbf{R}^{+}$action $\omega \mapsto \lambda \omega$, and let $\pi: C \rightarrow M$ be the projection. The Fefferman metric will be defined on the total space of $C$. 
To simplify the notation, we will usually use the same symbol to denote both a form on $M$ and its lift to $C$ (omitting the $\pi^{*}$ from the notation), making clear by the context which is meant.

The pseudohermitian structure induces a number of canonical differential forms on $C$, from which the metric will be constructed. First, since $K$ is a bundle of differential forms, it carries a natural tautologous $(n+1)$-form $\xi$, whose value at any point $\omega \in K$ is the lift to $K$ of $\omega$ itself. The pseudohermitian structure $\theta$ gives rise to a natural embedding $\iota_{\theta}: C \rightarrow K$, as follows. A point in $C$ is an equivalence class of $(n+1,0)$-forms under multiplication by positive reals. Choose a unique representative $\varsigma_{0}$ by means of the volume normalization

$$
\left.\left.i^{n^{2}} n ! \theta \wedge(T\rfloor \zeta_{0}\right) \wedge(T\rfloor \bar{\zeta}_{0}\right)=\theta \wedge d \theta^{n} .
$$

That this is possible is guaranteed by the following

(3.2) LEMMA. If $\omega$ is any smooth, nonvanishing $(n+1,0)$-form on $M$, then

$$
\left.\left.i^{n^{2}} n ! \theta \wedge(T\rfloor \omega\right) \wedge(T\rfloor \bar{\omega}\right)=\lambda \theta \wedge d \theta^{n}
$$

for some smooth, strictly positive function $\lambda$ on $M$.

ProOF. Only the positivity of $\lambda$ needs to be checked. We can choose an admissible coframe $\left\{\theta^{\alpha}\right\}$ such that $\left.T\right\rfloor \omega=\theta^{1} \wedge \cdots \wedge \theta^{n}$ and $d \theta=i h_{\alpha \bar{\beta}} \theta^{\alpha} \wedge \theta^{\bar{\beta}}$, where $\left(h_{\alpha \bar{\beta}}\right)$ is positive definite. A straightforward exercise in linear algebra then shows that

$$
\left.\left.d \theta^{n}=i^{n^{2}} n ! \operatorname{det}\left(h_{\alpha \bar{\beta}}\right)(T\rfloor \omega\right) \wedge(T\rfloor \bar{\omega}\right) .
$$

Since $\operatorname{det}\left(h_{\alpha \bar{\beta}}\right)>0$, wedging with $\theta$ completes the proof.

Thus, for any equivalence class $[\omega] \in C$, we set $\iota_{\theta}[\omega]=\varsigma_{0}$, where $\varsigma_{0}$ is the unique positive multiple of $\omega$ satisfying (3.1). This embedding allows us to pull the tautologous from $\xi$ back to $C$. Setting $\zeta=\iota_{\theta}^{*} \xi, \zeta$ is a globally defined $(n+1)$-form on $C$.

We also define a canonical vertical vector field $S$ on $C$ as the tangent to the natural $S^{1}$ action on $C,[\omega] \mapsto\left[e^{i \theta} \omega\right]$.

If $\varsigma_{0}$ is any $(n+1,0)$-form on (a subset of) $M$ satisfying the volume normalization (3.1), then we can lift $\zeta_{0}$ to $C$ and write $\zeta=e^{i \gamma} \zeta_{0}$, thereby defining $\gamma$ as a fiber coordinate on $C$. It is obvious that $d \gamma(S) \equiv 1$.

The major part of the Fefferman metric will be induced by the Levi form $L_{\theta}$. First, $L_{\theta}$ can be extended to a (degenerate) symmetric 2-tensor on all of $T M$, by requiring $L_{\theta}(T, V)=0$ for any $V \in T M$. We then lift $L_{\theta}$ to a tensor on $C$, still denoted by $L_{\theta}$, which is degenerate on the two-dimensional subspace of $T C$ spanned by $S$ and any lift of $T$.

Observe that, if $\sigma$ is any 1 -form such that $\sigma(S) \neq 0$, the symmetric 2-tensor $L_{\theta}+2 \theta \cdot \sigma$ is nondegenerate on $T C$ with Lorentz signature. If $\gamma$ is a local fiber coordinate for $C$ such that $d \gamma(S) \equiv 1$, an obvious choice for $\sigma$ would be a constant multiple of $d \gamma$; there is, however, nothing canonical about this choice. We are going to determine $\sigma$ uniquely by means of intrinsic normalizations on $C$ so that the resulting metric is conformally invariant under changes in $\theta$.

To describe the normalizations, we must introduce one more natural form on $C$. 
(3.3) LEMMA. There is a unique $n$-form $\eta$ on $C$ satisfying $\varsigma=\theta \wedge \eta, V\rfloor \eta=0$ for any lift $V$ of $T$.

Proof. Let $V$ be a lift to $C$ of $T$, and set $\eta=V\rfloor \zeta$. Since $S\rfloor \zeta=0$, this definition is independent of the choice of $V$, and $\eta$ is easily seen to satisfy the conclusions of the lemma.

We can now specify the 1-form $\sigma$ uniquely.

(3.4) PROPOSITION. There is a unique real 1 -form $\sigma$ on $C$ satisfying

$$
\begin{gathered}
d \varsigma=i(n+2) \sigma \wedge \varsigma \\
\sigma \wedge d \eta \wedge \bar{\eta}=(\operatorname{Tr} d \sigma) i \sigma \wedge \theta \wedge \eta \wedge \bar{\eta}
\end{gathered}
$$

PrOOF. Choose (locally) an $(n+1,0)$-form $\varsigma_{0}$ on $M$ satisfying (3.1), and write $\zeta=e^{i \gamma} \varsigma_{0}$, where $\gamma$ is a local fiber coordinate on $C$.

The integrability of the CR structure of $M$ implies that $d \varsigma_{0}=i(n+2) \sigma_{0} \wedge \varsigma_{0}$ for some 1 -form $\sigma_{0}$, which is determined only up to the addition of a $(1,0)$-form. If we require that $\sigma_{0}$ be real, then it is uniquely determined up to addition of a multiple of $\theta$. Thus on $C$

$$
\begin{aligned}
d \zeta & =e^{i \gamma}\left(i d \gamma \wedge \varsigma_{0}+i(n+2) \sigma_{0} \wedge \varsigma_{0}\right) \\
& =i(n+2)\left(\frac{1}{n+2} d \gamma+\sigma_{0}\right) \wedge \varsigma
\end{aligned}
$$

so $\sigma=d \gamma /(n+2)+\sigma_{0}$ satisfies (3.5) and is uniquely determined $\bmod \theta$. Observe that for any such $\sigma, d \sigma$ is a form on $M$, so $\operatorname{Tr} d \sigma$ makes sense.

To fix the multiple of $\theta$, we use the second normalization (3.6). Observe that if $\sigma^{\prime}=\sigma+f \theta$, then

$$
\begin{aligned}
\sigma^{\prime} \wedge d \eta \wedge \bar{\eta} & =\sigma \wedge d \eta \wedge \bar{\eta}+f \theta \wedge d \eta \wedge \bar{\eta} \\
& =\sigma \wedge d \eta \wedge \bar{\eta}-f(d \varsigma-d \theta \wedge \eta) \wedge \bar{\eta} \\
& =\sigma \wedge d \eta \wedge \bar{\eta}-i(n+2) f \sigma \wedge \theta \wedge \eta \wedge \bar{\eta}
\end{aligned}
$$

where the last equality follows because $d \theta \wedge \eta \wedge \bar{\eta}=0$, being a $(2 n+2)$-form on $C$ which annihilates $S$. On the other hand,

$$
\begin{gathered}
d \sigma^{\prime} \equiv d \sigma+f d \theta \quad(\bmod \theta), \quad \operatorname{Tr} d \sigma^{\prime}=\operatorname{Tr} d \sigma+n f \\
\left(\operatorname{Tr} d \sigma^{\prime}\right) i \sigma^{\prime} \wedge \theta \wedge \eta \wedge \bar{\eta}=(\operatorname{Tr} d \sigma) i \sigma \wedge \theta \wedge \eta \wedge \bar{\eta}+i n f \sigma \wedge \theta \wedge \eta \wedge \bar{\eta} .
\end{gathered}
$$

Since the two sides of (3.6) transform by different multiplies of the nonzero volume form if $\sigma \wedge \theta \wedge \eta \wedge \bar{\eta}$, there is a unique choice of $f$ such that $\sigma^{\prime}=\sigma+f \theta$ satisfies (3.6).

To see that $f$ is real, it suffices to show that $d \eta \wedge \bar{\eta}$ is a real multiple of $i \theta \wedge \eta \wedge \bar{\eta}$. Contracting (3.1) with $T$ and lifting to $C$ yields

$$
\left.\left.d \theta^{n}=i^{n^{2}} n ! \pi^{*}(T\rfloor \varsigma_{0}\right) \wedge \pi^{*}(T\rfloor \bar{\zeta}_{0}\right)=i^{n^{2}} n ! \eta \wedge \bar{\eta} .
$$

Differentiating this,

$$
0=i^{n^{2}} n !\left(d \eta \wedge \bar{\eta}+(-1)^{n} \eta \wedge d \bar{\eta}\right)
$$

which says that $i^{n^{2}} d \eta \wedge \bar{\eta}$ is pure imaginary. On the other hand, it is immediate that $i^{n^{2}}(i \theta \wedge \eta \wedge \bar{\eta})$ is imaginary, so we are done. 
Now define the metric $g$ on $C$ by

$$
g=L_{\theta}+2 \theta \cdot \sigma \text {. }
$$

It is clear from the discussion above that $g$ is a nondegenerate metric with Lorentz signature, and is uniquely determined by the pseudohermitian structure $\theta$.

Our main result is

(3.8) THEOREM. If $\tilde{\theta}=e^{2 f} \theta$ is another pseudohermitian structure on $M$, and $\tilde{g}$ is the associated Lorentz metric, then $\tilde{g}=e^{2 f} g$. Thus the conformal class of $g$ is a $C R$ invariant of $M$.

This can be proved directly by deriving the transformation law for $\sigma$ from (3.5) and (3.6). However, we defer the proof until $\S 5$, where we will be able to give a simpler proof.

It is worth noting that the two normalizations (3.5) and (3.6) correspond directly to the first two approximations to the solution of the Monge-Ampère equation usd by Fefferman when he originally defined the metric. This will become clear in $\S 5$, when the intrinsically defined metric $g$ is related to Fefferman's extrinsic definition.

4. The Webster-Stanton connection. In [12], S. Webster showed there is a natural connection in the bundle $T_{1,0}$ adapted to a pseudohermitian structure. This was subsequently extended to a connection to CTM by C. Stanton [11]. In this section we recall the definition and main properties of the connection.

To define the connection, choose an admissible coframe $\left\{\theta^{\alpha}\right\}$ and dual frame $\left\{Z_{\alpha}\right\}$ for $T_{1,0}$. Webster showed that there are uniquely determined 1-forms $\omega_{\alpha}^{\beta}, \tau^{\beta}$ on $M$ satisfying

$$
\begin{gathered}
d \theta^{\beta}=\theta^{\alpha} \wedge \omega_{\alpha}^{\beta}+\theta \wedge \tau^{\beta}, \\
\omega_{\alpha \bar{\beta}}+\omega_{\bar{\beta} \alpha}=d h_{\alpha \bar{\beta}}, \\
\tau_{\alpha} \wedge \theta^{\alpha}=0,
\end{gathered}
$$

in which we have used the matrix $h_{\alpha \bar{\beta}}$ to raise and lower indices, e.g. $\omega_{\alpha \bar{\beta}}=\omega_{\alpha}^{\gamma} h_{\gamma \bar{\beta}}$. By (4.3), we can write

$$
\tau_{\alpha}=A_{\alpha \gamma} \theta^{\gamma}
$$

with $A_{\alpha \gamma}=A_{\gamma \alpha}$.

Covariant differentiation is defined by

$$
D Z_{\alpha}=\omega_{\alpha}^{\beta} \otimes Z_{\beta}, \quad D Z_{\bar{\alpha}}=\omega_{\bar{\alpha}}^{\bar{\beta}} \otimes Z_{\bar{\beta}}, \quad D T=0 .
$$

For a function $f$ on $M$, we will write $f_{\alpha}=Z_{\alpha} f, f_{\bar{\alpha}}=Z_{\bar{\alpha}} f, f_{0}=T f$, so that $D f=d f=f_{\alpha} \theta^{\alpha}+f_{\bar{\alpha}} \theta^{\bar{\alpha}}+f_{0} \theta$. The second covariant differential $D^{2} f$ of $f$ is the 2-tensor with components

$$
\begin{aligned}
& f_{\alpha \beta}=\overline{\overline{f_{\bar{\alpha} \bar{\beta}}}}=Z_{\beta} Z_{\alpha} f-\omega_{\alpha}^{\gamma}\left(Z_{\beta}\right) Z_{\gamma} f, \quad f_{\alpha \bar{\beta}}=\overline{\bar{f}}_{\bar{\alpha} \beta}=Z_{\bar{\beta}} Z_{\alpha} f-\omega_{\alpha}^{\gamma}\left(Z_{\bar{\beta}}\right) Z_{\gamma} f, \\
& f_{0 \alpha}=\overline{\bar{f}_{0 \bar{\alpha}}}=Z_{\alpha} T f, \quad f_{\alpha 0}=\overline{\bar{f}}_{\bar{\alpha} 0}=T Z_{\alpha} f-\omega_{\alpha}^{\gamma}(T) Z_{\gamma} f, \quad f_{00}=T^{2} f .
\end{aligned}
$$

Observe that (2.2) and (4.1) imply

$$
\begin{aligned}
{\left[Z_{\bar{\beta}}, Z_{\alpha}\right] } & =i h_{\alpha \bar{\beta}} T+\omega_{\alpha}^{\gamma}\left(Z_{\bar{\beta}}\right) Z_{\gamma}-\omega_{\bar{\beta}}^{\bar{\gamma}}\left(Z_{\alpha}\right) Z_{\bar{\gamma}}, \\
{\left[Z_{\beta}, Z_{\alpha}\right] } & =\omega_{\alpha}{ }^{\gamma}\left(Z_{\beta}\right) Z_{\gamma}-\omega_{\beta}{ }^{\gamma}\left(Z_{\alpha}\right) Z_{\gamma}, \\
{\left[Z_{\alpha}, T\right] } & =A^{\bar{\gamma}_{\alpha}} Z_{\bar{\gamma}}-\omega_{\alpha}{ }^{\gamma}(T) Z_{\gamma},
\end{aligned}
$$


and therefore

$$
f_{\alpha \bar{\beta}}-f_{\bar{\beta} \alpha}=i h_{\alpha \bar{\beta}} f_{0}, \quad f_{\alpha \beta}-f_{\beta \alpha}=0, \quad f_{0 \alpha}-f_{\alpha 0}=A_{\alpha}^{\bar{\gamma}_{\alpha}} f_{\bar{\gamma}} .
$$

In general, if $T_{\alpha_{1} \cdots \alpha_{k}}^{\beta_{1} \cdots \beta_{l}}$ are the components of a tensor of degree $(k, l)$, its $r$ th covariant derivative $D^{r} T$ is the tensor of degree $(k+r, l)$ with components which we shall denote $T_{\alpha_{1} \cdots \alpha_{k}, \gamma_{1} \cdots \gamma_{r}}^{\beta_{1} \cdots \beta_{l}}$.

The curvature of the Webster-Stanton connection, expressed in terms of the coframe $\left\{\theta=\theta^{0}, \theta^{\alpha}, \theta^{\bar{\alpha}}\right\}$, is

$$
\begin{aligned}
& \Pi_{\beta}^{\alpha}=\overline{\Pi_{\bar{\beta}}{ }^{\bar{\alpha}}}=d \omega_{\beta}{ }^{\alpha}-\omega_{\beta}^{\gamma} \wedge \omega_{\gamma}{ }^{\alpha}, \\
& \Pi_{0}{ }^{\alpha}=\Pi_{\alpha}{ }^{0}=\Pi_{0}{ }^{\bar{\beta}}=\Pi_{\bar{\beta}^{0}}{ }^{0}=\Pi_{0}{ }^{0}=0 .
\end{aligned}
$$

Webster showed that $\Pi_{\beta}^{\alpha}$ can be written

$$
\Pi_{\beta}{ }^{\alpha}=R_{\beta}{ }_{\rho \bar{\rho}} \theta^{\rho} \wedge \theta^{\bar{\sigma}}+W_{\beta}{ }^{\alpha}{ }_{\rho} \theta^{\rho} \wedge \theta-W^{\alpha}{ }_{\beta \bar{\rho}} \theta^{\bar{\rho}} \wedge \theta+i \theta_{\beta} \wedge \tau^{\alpha}-i \tau_{\beta} \wedge \theta^{\alpha}
$$

where the coefficients satisfy

$$
R_{\beta \bar{\alpha} \rho \bar{\sigma}}=\overline{R_{\alpha \bar{\beta} \sigma \bar{\rho}}}=R_{\bar{\alpha} \beta \bar{\sigma} \rho}=R_{\rho \bar{\alpha} \beta \bar{\sigma}}, \quad W_{\beta \bar{\alpha} \gamma}=W_{\gamma \bar{\alpha} \beta} .
$$

The Webster-Ricci tensor of $(M, \theta)$ is the hermitian form $\rho$ on $T_{1,0}$ defined by

$$
\rho(X, \bar{Y})=R_{\alpha \bar{\beta}} X^{\alpha} Y^{\bar{\beta}}
$$

where $X=X^{\alpha} Z_{\alpha}, Y=Y^{\beta} Z_{\beta}, R_{\alpha \bar{\beta}}=R_{\gamma}{ }^{\gamma}{ }_{\alpha \bar{\beta}}$. The Webster scalar curvature is

$$
R=R_{\alpha}^{\alpha}=h^{\alpha \bar{\beta}} R_{\alpha \bar{\beta}}
$$

Observe that this can also be written

$$
R=\operatorname{Tr} i \Pi_{\alpha}^{\alpha}=\operatorname{Tr} i d \omega_{\alpha}^{\alpha} .
$$

The sublaplacian has a particularly simple expression in terms of covariant derivatives.

(4.10) Proposition. If $u \in C^{\infty}(M), \Delta_{b} u=-\left(u_{\alpha}{ }^{\alpha}+u_{\bar{\alpha}}{ }^{\bar{\alpha}}\right)$.

ProOF. If $v \in C_{c}^{\infty}(M)$, then from (2.4) and (2.5) we have

$$
\begin{aligned}
\left\langle\Delta_{b} u, \bar{v}\right\rangle_{\theta} & =i n \int_{M} \theta \wedge \partial_{b} u \wedge \bar{\partial}_{b} \bar{v} \wedge d \theta^{n-1}+i n \int_{M} \theta \wedge \partial_{b} \bar{v} \wedge \bar{\partial}_{b} u \wedge d \theta^{n-1} \\
& =i n \int_{M} \bar{v} \theta \wedge d \partial_{b} u \wedge d \theta^{n-1}-i n \int_{M} \bar{v} \theta \wedge d \bar{\partial}_{b} u \wedge d \theta^{n-1}
\end{aligned}
$$

It is easy to check that if $\omega$ is any 2 -form,

$$
n \theta \wedge \omega \wedge d \theta^{n-1}=(\operatorname{Tr} \omega) \theta \wedge d \theta^{n}
$$

and thus

Since

$$
\begin{aligned}
\left\langle\Delta_{b} u, \bar{v}\right\rangle_{\theta} & =\int_{M} \bar{v}\left(\operatorname{Tr} i d \partial_{b} u-\operatorname{Tr} i d \bar{\partial}_{b} u\right) \psi \\
& =\left\langle\operatorname{Tr} i d \partial_{b} u-\operatorname{Tr} i d \bar{\partial}_{b} u, \bar{v}\right\rangle_{\theta}
\end{aligned}
$$

$$
i d \partial_{\beta} u=i d\left(u_{\alpha} \theta^{\alpha}\right) \equiv-i u_{\alpha \bar{\beta}} \theta^{\alpha} \wedge \theta^{\bar{\beta}} \quad\left(\bmod \theta^{\alpha} \wedge \theta^{\gamma}, \theta^{\bar{\alpha}} \wedge \theta^{\bar{\gamma}}, \theta\right),
$$

it follows that $\operatorname{Tr} i d \partial_{b} u=-u_{\alpha}{ }^{\alpha}$, and similarly $-\operatorname{Tr} i d \bar{\partial}_{b} u=-u_{\bar{\alpha}}{ }^{\bar{\alpha}}$. Thus $\Delta_{b} u=$ $-\left(u_{\alpha}{ }^{\alpha}+u_{\bar{\alpha}}{ }^{\bar{\alpha}}\right)$ as claimed. 
5. The Fefferman metric: second characterization. Using the WebsterStanton connection, we now give a second intrinsic characterization of the Fefferman metric associated with a pseudohermitian structure $\theta$. Let $C=K^{*} / \mathbf{R}^{+}$be the circle bundle over $M$ defined in $\S 3$. As in that section we will use a single symbol to denote both a form on $M$ and its lift to $C$.

Choose an admissible coframe $\left\{\theta^{\alpha}\right\}$ over an open set $U \subset M$. Observe that

$$
\varsigma_{0}=\left(\operatorname{det} h_{\alpha \bar{\beta}}\right)^{1 / 2} \theta \wedge \theta^{1} \wedge \cdots \wedge \theta^{n}
$$

is a nonvanishing $(n+1,0)$-form over $U$ which satisfies the volume normalization (3.1). Lifting $\varsigma_{0}$ to $C$, we can write the intrinsic $(n+1)$-form $\zeta$ on $C$ as $\zeta=e^{i \gamma} \zeta_{0}$, thus defining $\gamma$ as a local fiber coordinate on $C$.

(5.1) THEOREM. The Lorentz metric $g$ on $C$ defined by (3.7) is also given by

$$
g=\theta^{\alpha} \cdot \theta_{\alpha}+2 \theta \cdot \sigma
$$

in which

$$
\sigma=\frac{1}{n+2}\left(d \gamma+i \omega_{\alpha}^{\alpha}-\frac{i}{2} h^{\alpha \bar{\beta}} d h_{\alpha \bar{\beta}}-\frac{1}{2(n+1)} R \theta\right)
$$

Thus (5.2) and (5.3) are independent of the choice of admissible coframe $\left\{\theta^{\alpha}\right\}$, and are globally defined on $M$.

PROOF. It is immediate that $L_{\theta}=\theta^{\alpha} \cdot \theta_{\alpha}$. Contracting (4.2) with $i h^{\alpha \bar{\beta}}$, we see that $i \omega_{\alpha}{ }^{\alpha}-(i / 2) h^{\alpha \bar{\beta}} d h_{\alpha \bar{\beta}}$ is real, and so $\sigma$ is real. So we need only verify that $\sigma$, defined by (5.3), satisfies the normalizations (3.5) and (3.6).

We compute

$$
\begin{gathered}
d \varsigma=i d \gamma \wedge \varsigma+e^{i \gamma} d \varsigma_{0} \\
d \zeta_{0}=\frac{1}{2}\left(\operatorname{det} h_{\alpha \bar{\beta}}\right)^{1 / 2} h^{\alpha \bar{\beta}} d h_{\alpha \bar{\beta}} \wedge \theta \wedge \theta^{1} \wedge \cdots \wedge \theta^{n} \\
+\left(\operatorname{det} h_{\alpha \bar{\beta}}\right)^{1 / 2} \sum_{\alpha}(-1)^{\alpha} \theta \wedge \theta^{1} \wedge \cdots \wedge d \theta^{\alpha} \wedge \cdots \wedge \theta^{n} \\
=\left(\frac{1}{2} h^{\alpha \bar{\beta}} d h_{\alpha \bar{\beta}}-\omega_{\alpha}^{\alpha}\right) \wedge \varsigma_{0} .
\end{gathered}
$$

On the other hand,

$$
i(n+2) \sigma \wedge \varsigma=\left(i d \gamma-\omega_{\alpha}^{\alpha}+\frac{1}{2} h^{\alpha \bar{\beta}} d h_{\alpha \bar{\beta}}\right) \wedge \varsigma
$$

so $\sigma$ satisfies (3.5).

Next observe that $\eta=\left(\operatorname{det} h_{\alpha \bar{\beta}}\right)^{1 / 2} e^{i \gamma} \theta \wedge \theta^{1} \wedge \cdots \wedge \theta^{n}$, and

$$
\begin{aligned}
d \eta & \equiv i\left(d \gamma+i \omega_{\alpha}{ }^{\alpha}-\frac{i}{2} h^{\alpha \bar{\beta}} d h_{\alpha \bar{\beta}}\right) \wedge \eta \quad\left(\bmod \theta^{\bar{\gamma}}\right) \\
\sigma \wedge d \eta \wedge \bar{\eta}= & \frac{1}{n+2}\left(d \gamma+i \omega_{\alpha}{ }^{\alpha}-\frac{i}{2} h^{\alpha \bar{\beta}} d h_{\alpha \bar{\beta}}-\frac{1}{2(n+1)} R \theta\right) \\
& \wedge i\left(d \gamma+i \omega_{\alpha}^{\alpha}-\frac{i}{2} h^{\alpha \bar{\beta}} d h_{\alpha \bar{\beta}}\right) \wedge \eta \wedge \bar{\eta} \\
= & -\frac{i}{2(n+1)(n+2)} R \theta \wedge d \gamma \wedge \eta \wedge \bar{\eta}
\end{aligned}
$$


On the other hand, using (4.9) and the fact that $d\left(h^{\alpha \bar{\beta}} d h_{\alpha \bar{\beta}}\right)=d^{2} \log \left(\operatorname{det} h_{\alpha \bar{\beta}}\right)=0$,

$$
\begin{aligned}
& d \sigma=\frac{1}{n+2}\left(i d \omega_{\alpha}{ }^{\alpha}-\frac{1}{2(n+1)} R d \theta-\frac{1}{2(n+1)} d R \wedge \theta\right), \\
& \operatorname{Tr} d \sigma=\frac{1}{n+2} \operatorname{Tr} i d \omega_{\alpha}{ }^{\alpha}-\frac{n}{2(n+1)(n+2)} R=\frac{1}{2(n+1)} R .
\end{aligned}
$$

Thus

$$
(\operatorname{Tr} d \sigma) i \sigma \wedge \theta \wedge \eta \wedge \bar{\eta}=\frac{1}{2(n+1)(n+2)} \operatorname{Rid} \gamma \wedge \theta \wedge \eta \wedge \bar{\eta}
$$

Comparing this with (5.4) completes the proof.

The next step is to examine how the metric $g$, defined by either (3.7) or (5.2), transforms under a change of pseudohermitian structure. First we must determine the transformation law for the connection forms. Let $\tilde{\theta}=e^{2 f} \theta$ be a new choice of pseudohermitian structure for $M$. Choose an admissible coframe $\left\{\theta^{\alpha}\right\}$ for $\theta$, with dual frame $\left\{Z_{\alpha}\right\}$. Recall the notation $f_{\alpha}, f_{\alpha \bar{\beta}}$, etc., for the covariant derivatives of $f$, described in $\S 4$. All covariant derivatives will be taken with respect to the coframe $\left\{\theta^{\alpha}\right\}$ and the pseudohermitian structure $\theta$.

One checks easily that

$$
\tilde{\theta}^{\alpha}=e^{f}\left(\theta^{\alpha}+2 i f^{\alpha} \theta\right)
$$

satisfies

$$
i h_{\alpha \bar{\beta}} \tilde{\theta}^{\alpha} \wedge \tilde{\theta}^{\bar{\beta}}=e^{2 f}(d \theta+2 d f \wedge \theta)=d \tilde{\theta},
$$

and thus $\left\{\tilde{\theta}^{\alpha}\right\}$ is an admissible coframe for $\tilde{\theta}$, with the same matrix $\tilde{h}_{\alpha \bar{\beta}}=h_{\alpha \bar{\beta}}$. We will compute the connection forms in terms of the coframe $\left\{\tilde{\theta}^{\alpha}\right\}$. Observe that, for any 2-form $\omega, \widetilde{\operatorname{Tr}} \omega=e^{-2 f} \operatorname{Tr} \omega$.

(5.6) LEMMA. With

$$
\begin{gathered}
\tilde{\omega}_{\beta}{ }^{\alpha}=\omega_{\beta}{ }^{\alpha}+2\left(f_{\beta} \theta^{\alpha}-f^{\alpha} \theta_{\beta}\right)+\delta_{\beta}^{\alpha}\left(f_{\gamma} \theta^{\gamma}-f^{\gamma} \theta_{\gamma}\right) \\
+i\left(f^{\alpha}{ }_{\beta}+f_{\beta}{ }^{\alpha}+4 f_{\beta} f^{\alpha}+4 \delta_{\beta}^{\alpha} f_{\gamma} f^{\gamma}\right) \theta \\
\tilde{\tau}_{\alpha}=\tilde{A}_{\alpha \beta} \tilde{\theta}^{\beta}
\end{gathered}
$$

where

$$
\tilde{A}_{\alpha \beta}=e^{-2 f}\left(A_{\alpha \beta}+2 i f_{\alpha \beta}-4 i f_{\alpha} f_{\beta}\right)
$$

we have

$$
\begin{gathered}
d \tilde{\theta}^{\alpha}=\tilde{\theta}^{\beta} \wedge \tilde{\omega}_{\beta}{ }^{\alpha}+\tilde{\theta} \wedge \tilde{\tau}^{\alpha}, \\
\tilde{\omega}_{\alpha \bar{\beta}}+\tilde{\omega}_{\bar{\beta} \alpha}=d h_{\alpha \bar{\beta}}, \\
\tilde{\tau}^{\alpha} \wedge \tilde{\theta}_{\alpha}=0
\end{gathered}
$$

and therefore $\tilde{\omega}_{\beta}{ }^{\alpha}, \tilde{\tau}^{\alpha}$ are the Webster connection forms for $\tilde{\theta}$.

PROOF. First, an easy calculation shows that $\tilde{\omega}_{\beta}{ }^{\alpha}$ and $\tilde{\tau}^{\alpha}$, defined by (5.7) and (5.8), satisfy (5.11) and (5.12). Differentiating (5.5),

$$
\begin{aligned}
d \tilde{\theta}^{\alpha}=e^{f}\left(f_{\beta} \theta^{\beta} \wedge \theta^{\alpha}+f_{\bar{\beta}} \theta^{\bar{\beta}} \wedge \theta^{\alpha}+f_{0} \theta \wedge \theta^{\alpha}+2 i f_{\beta} f^{\alpha} \theta^{\beta} \wedge \theta\right. & \\
+2 i f_{\bar{\beta}} f^{\alpha} \theta^{\bar{\beta}} \wedge \theta+\theta^{\beta} \wedge \omega_{\beta}{ }^{\alpha}+\theta & \wedge \tau^{\alpha}+2 i Z_{\beta} f^{\alpha} \theta^{\beta} \wedge \theta \\
& \left.+2 i Z_{\bar{\beta}} f^{\alpha} \theta^{\bar{\beta}} \wedge \theta-2 f^{\alpha} \theta^{\beta} \wedge \theta_{\beta}\right) .
\end{aligned}
$$


On the other hand,

$$
\begin{array}{r}
\tilde{\theta}^{\beta} \wedge \tilde{\omega}_{\beta}{ }^{\alpha} \equiv e^{f}\left(\theta^{\beta} \wedge \omega_{\beta}{ }^{\alpha}+2 f_{\beta} \theta^{\beta} \wedge \theta^{\alpha}-2 f^{\alpha} \theta^{\beta} \wedge \theta_{\beta}+f_{\gamma} \theta^{\alpha} \wedge \theta^{\gamma}-f^{\gamma} \theta^{\alpha} \wedge \theta_{\gamma}\right. \\
+i\left(f^{\alpha}{ }_{\beta}+f_{\beta}{ }^{\alpha}\right) \theta^{\beta} \wedge \theta+4 i f_{\beta} f^{\alpha} \theta^{\beta} \wedge \theta+4 i f_{\gamma} f^{\gamma} \theta^{\alpha} \wedge \theta \\
\left.+2 i f^{\beta} \theta \wedge \omega_{\beta}{ }^{\alpha}+4 i f^{\beta} f_{\beta} \theta \wedge \theta^{\alpha}+2 i f^{\alpha} f_{\gamma} \theta \wedge \theta^{\gamma}\right) \\
\bmod \theta \wedge \theta^{\bar{\gamma}} \\
\equiv e^{f}\left(\theta^{\beta} \wedge \omega_{\beta}^{\alpha}+f_{\beta} \theta^{\beta} \wedge \theta^{\alpha}-2 f^{\alpha} \theta^{\beta} \wedge \theta_{\beta}+f_{\bar{\gamma}} \theta^{\bar{\gamma}} \wedge \theta^{\alpha}+2 i f^{\alpha}{ }_{\beta} \theta^{\beta} \wedge \theta\right. \\
\left.-f_{0} \theta^{\alpha} \wedge \theta+2 i f_{\beta} f^{\alpha} \theta^{\beta} \wedge \theta-2 i f^{\beta} \omega_{\beta}{ }^{\alpha}\left(Z_{\gamma}\right) \theta^{\gamma} \wedge \theta\right) \\
\bmod \theta \wedge \theta^{\bar{\gamma}}
\end{array}
$$

where we have used (4.6), and the fact that $f^{\alpha}{ }_{\beta}=Z_{\beta} f^{\alpha}+f^{\gamma} \omega_{\gamma}{ }^{\alpha}\left(Z_{\beta}\right)$, which follows from (4.2).

To prove (5.10), therefore, it suffices to show that $\tilde{A}_{\bar{\beta}}^{\alpha}$, defined by (5.9), satisfies

$$
\tilde{A}_{\bar{\beta}}^{\alpha}=2 d \tilde{\theta}^{\alpha}\left(\tilde{T} \wedge \tilde{Z}_{\bar{\beta}}\right)
$$

We observe first that

$$
\tilde{T}=e^{-2 f}\left(T+2 i f^{\bar{\gamma}} Z_{\bar{\gamma}}-2 i f^{\gamma} Z_{\gamma}\right), \quad \tilde{Z}_{\bar{\beta}}=e^{-f} Z_{\bar{\beta}},
$$

and so, using (5.13),

$$
\begin{aligned}
2 d \tilde{\theta}^{\alpha} & \left(\tilde{T} \wedge \tilde{Z}_{\bar{\beta}}\right)=2 e^{-3 f} d \tilde{\theta}^{\alpha}\left(T \wedge Z_{\bar{\beta}}+2 i f^{\bar{\gamma}} Z_{\bar{\gamma}} \wedge Z_{\bar{\beta}}-2 i f^{\gamma} Z_{\gamma} \wedge Z_{\bar{\beta}}\right) \\
& =e^{-2 f}\left(2 i f^{\alpha} f_{\bar{\beta}}-2 i f_{\bar{\beta}} f^{\alpha}-2 i f^{\gamma} \omega_{\gamma}{ }^{\alpha}\left(Z_{\bar{\beta}}\right)+\tau^{\alpha}\left(Z_{\bar{\beta}}\right)-2 i Z_{\bar{\beta}} f^{\alpha}+4 i f^{\alpha} f_{\bar{\beta}}\right) \\
& =e^{-2 f}\left(A_{\bar{\beta}}^{\alpha}-2 i f_{\bar{\beta}}^{\alpha}+4 i f^{\alpha} f_{\bar{\beta}}\right)=\tilde{A}_{\bar{\beta}}^{\alpha},
\end{aligned}
$$

which proves (5.10).

Next we calculate the transformation law for the Webster scalar curvature $R$.

(5.15) PROPOSITION. The Webster scalar curvature $\tilde{R}$ associated with $\tilde{\theta}=$ $e^{2 f} \theta$ is

$$
\tilde{R}=e^{-2 f}\left(R+2(n+1) \Delta_{b} f-4 n(n+1) f_{\alpha} f^{\alpha}\right) .
$$

ProOF. From (5.7),

$$
\begin{aligned}
& \tilde{\omega}_{\alpha}^{\alpha}=\omega_{\alpha}^{\alpha}+(n+2)\left(f_{\alpha} \theta^{\alpha}-f_{\bar{\beta}} \theta^{\bar{\beta}}\right)+i\left(f_{\bar{\beta}}{ }^{\bar{\beta}}+f_{\alpha}{ }^{\alpha}+4(n+1) f_{\alpha} f^{\alpha}\right) \theta, \\
& d \tilde{\omega}_{\alpha}^{\alpha} \equiv d \omega_{\alpha}^{\alpha}+(n+2)\left(f_{\alpha \bar{\beta}} \theta^{\bar{\beta}} \wedge \theta^{\alpha}-f_{\bar{\beta} \alpha} \theta^{\alpha} \wedge \theta^{\bar{\beta}}\right) \\
& +i\left(f_{\bar{\beta}}^{\bar{\beta}}+f_{\alpha}^{\alpha}+4(n+1) f_{\alpha} f^{\alpha}\right) d \theta \quad\left(\bmod \theta^{\alpha} \wedge \theta^{\gamma}, \theta^{\bar{\alpha}} \wedge \theta^{\bar{\gamma}}, \theta\right), \\
& \tilde{R}=\widetilde{\operatorname{Tr}} i d \tilde{\omega}_{\alpha}{ }^{\alpha}=e^{-2 f}\left(R-(n+2)\left(f_{\alpha}{ }^{\alpha}+f_{\bar{\beta}}{ }^{\bar{\beta}}\right)-n\left(f_{\bar{\beta}}{ }^{\bar{\beta}}+f_{\alpha}{ }^{\alpha}+4(n+1) f_{\alpha} f^{\alpha}\right)\right) \\
& =e^{-2 f}\left(R+2(n+1) \Delta_{b} f-4 n(n+1) f_{\alpha} f^{\alpha}\right) \text {. }
\end{aligned}
$$

The transformation law for $\sigma$ is now easy to compute. 
(5.16) PROPOSITION. With $\tilde{\theta}=e^{2 f} \theta$ as above, the 1-form $\tilde{\sigma}$ associated with $\tilde{\theta}$ is given by

$$
\tilde{\sigma}=\sigma+i\left(f_{\alpha} \theta^{\alpha}-f_{\bar{\beta}} \theta^{\bar{\beta}}\right)-2 f_{\alpha} f^{\alpha} \theta .
$$

PROOF. Using formula (5.5) for $\tilde{\theta}^{\alpha}$, the $(n+1,0)$-form

$$
\tilde{\zeta}_{0}=\left(\operatorname{det} h_{\alpha \bar{\beta}}\right)^{1 / 2} \tilde{\theta} \wedge \tilde{\theta}^{1} \wedge \cdots \wedge \tilde{\theta}^{n}=e^{(n+2) f_{\varsigma_{0}}}
$$

satisfies the volume normalization (3.1) for $\tilde{\theta}$, and thus the canonical $(n+1)$-form $\tilde{\zeta}$ on $C$ associated with $\tilde{\theta}$ is

$$
\tilde{\zeta}=e^{i \gamma} \tilde{\zeta}_{0}=e^{(n+2) f} \zeta,
$$

where $\gamma$ is the same fiber coordinate as before. Thus

$$
\begin{aligned}
\tilde{\sigma}= & \frac{1}{n+2}\left(d \gamma+i \tilde{\omega}_{\alpha}^{\alpha}-\frac{i}{2} h^{\alpha \bar{\beta}} d h_{\alpha \bar{\beta}}-\frac{1}{2(n+1)} \tilde{R} \tilde{\theta}\right) \\
= & \frac{1}{n+2}\left(d \gamma+i \omega_{\alpha}^{\alpha}+i(n+2)\left(f_{\alpha} \theta^{\alpha}-f_{\bar{\beta}} \theta^{\bar{\beta}}\right)-\left(f_{\alpha}{ }^{\alpha}+f_{\bar{\beta}} \bar{\beta}+4(n+1) f_{\alpha} f^{\alpha}\right) \theta\right. \\
& \left.\quad-\frac{i}{2} h^{\alpha \bar{\beta}} d h_{\alpha \bar{\beta}}-\frac{1}{2(n+1)}\left(R+2(n+1) \Delta_{b} f-4 n(n+1) f_{\alpha} f^{\alpha}\right) \theta\right) \\
= & \sigma+i\left(f_{\alpha} \theta^{\alpha}-f_{\bar{\beta}} \theta^{\bar{\beta}}\right)-2 f_{\alpha} f^{\alpha} \theta . \quad
\end{aligned}
$$

(5.17) THEOREM. If $\tilde{g}$ is the Lorentz metric induced by $\tilde{\theta}=e^{2 f} \theta$, then $\tilde{g}=$ $e^{2 f} g$. Thus the conformal class of $g$ is a CR invariant of $M$.

ProOF. By Proposition (5.16),

$$
\begin{aligned}
\tilde{g} & =\tilde{\theta}^{\alpha} \cdot \tilde{\theta}_{\alpha}+2 \tilde{\theta} \cdot \tilde{\sigma} \\
& =e^{2 f}\left(\theta^{\alpha} \cdot \theta_{\alpha}+2 i f^{\alpha} \theta \cdot \theta_{\alpha}-2 i f_{\alpha} \theta \cdot \theta^{\alpha}+4 f^{\alpha} f_{\alpha} \theta^{2}\right. \\
& \left.\quad+2 \theta \cdot \sigma+2 i f_{\alpha} \theta \cdot \theta^{\alpha}-2 i f_{\bar{\beta}} \theta \cdot \theta^{\bar{\beta}}-4 f_{\alpha} f^{\alpha} \theta^{2}\right) \\
& =e^{2 f} g . \quad \square
\end{aligned}
$$

It remains to compare the metric $g$ with the metric $G$ defined extrinsically for a hypersurface $M$ in $\mathbf{C}^{n+1}$ by Fefferman in [5]. Since both $G$ and $g$ are well defined up to a conformal factor, it suffices to compare $G$ and $g$ for one particular choice of pseudohermitian structure $\theta$.

Suppose $M$ is embedded as a hypersurface in $\mathbf{C}^{n+1}$. The restriction of the canonical bundle $K_{\mathrm{C}^{n+1}}$ to $M$ is naturally isomorphic to the canonical bundle $K$ of $M$, and the section $d z^{1} \wedge \cdots \wedge d z^{n+1}$ of $K_{\mathbf{C}^{n+1}}$ restricts to a nonvanishing section $\zeta_{0}$ of $K$. Writing any section of $K_{\mathbf{C}^{n+1}}$ as $z^{0} d z^{1} \wedge \cdots \wedge d z^{n+1}$ defines $z^{0}$ as a holomorphic fiber coordinate on $K_{\mathrm{C}^{n+1}}$. Then $\gamma=\arg z^{0}$ gives a fiber coordinate on the circle bundle $K_{\mathbf{C}^{n+1}}^{*} / \mathbf{R}^{+}$, which restricts to a fiber coordinate on $C=K^{*} / \mathbf{R}^{+}$.

Fefferman demonstrated the existence of a smooth defining function $u$ for $M$ which satisfies the complex Monge-Ampère equation

$$
J(u)=(-1)^{n+1} \operatorname{det}\left[\begin{array}{cc}
u & \partial u / \partial z^{\bar{k}} \\
\partial u / \partial z^{j} & \partial^{2} u / \partial z^{j} \partial z^{\bar{k}}
\end{array}\right]=1
$$


to second order along $M$. The Fefferman metric of $M$ is then the Lorentz metric on $C$ given by

$$
G=\frac{\partial^{2} u}{\partial z^{j} \partial z^{\bar{k}}} d z^{j} \cdot d z^{\bar{k}}+\frac{i}{n+2}(\bar{\partial} u-\partial u) \cdot d \gamma
$$

which obviously depends only on the second-order jet of $u$ along $M$.

We give $M$ the pseudohermitian structure defined by $\theta=i(\bar{\partial} u-\partial u) / 2$, and let $g$ be the Lorentz metric on $C$ induced by $\theta$, defined by (3.7).

(5.19) ThEOREM. $G=g$.

PROOF. A calculation shows that the $(n+1,0)$-form $\zeta_{0}=d z^{1} \wedge \cdots \wedge d z^{n+1}$ satisfies the volume normalization (3.1) with respect to $\theta$ if and only if $u$ satisfies (5.18) along $M$ (cf. [4]). F. Farris showed in [4] that the Lorentz metric

$$
g_{0}=L_{\theta}+\frac{2}{n+2} \theta \cdot d \gamma+\frac{1}{n+1} \alpha_{\varsigma 0} \theta^{2}
$$

in which $\alpha_{\varsigma_{0}} \in C^{\infty}(M)$ is defined by

$$
\left.\left.\left.\left.d(T\rfloor \varsigma_{0}\right) \wedge(T\rfloor \bar{\zeta}_{0}\right)=i \alpha_{\varsigma_{0}} \theta \wedge(T\rfloor \zeta_{0}\right) \wedge(T\rfloor \bar{\zeta}_{0}\right),
$$

agrees with the Fefferman metric $G$. Thus to prove that $g=G$ it suffices to show that

$$
\sigma=\frac{1}{n+2} d \gamma+\frac{1}{2(n+1)} \alpha_{\zeta 0} \theta
$$

for this choice of $\theta$.

Writing the canonical $(n+1)$-form on $C$ as $\zeta=e^{i \gamma} \zeta_{0}$, and using the fact that $\zeta_{0}$ is closed, we have $d \zeta=i d \gamma \wedge \zeta$. Thus (5.21) satisfies the first normalization (3.5). To see that it also satisfies the second, we calculate:

$$
\begin{aligned}
\operatorname{Tr} d \sigma & \left.=\frac{n}{2(n+1)} \alpha_{\varsigma_{0}}, \quad \eta=e^{i \gamma}(T\rfloor \varsigma_{0}\right), \\
d \eta & \left.\left.=e^{i \gamma}\left(i d \gamma \wedge(T\rfloor \varsigma_{0}\right)+d(T\rfloor \varsigma_{0}\right)\right),
\end{aligned}
$$

and therefore the second normalization (3.6) for this $\sigma$ is equivalent to

$$
\begin{aligned}
& \left.\left.\frac{n i}{2(n+1)(n+2)} \alpha_{\zeta_{0}} d \gamma \wedge \theta \wedge(T\rfloor \varsigma_{0}\right) \wedge(T\rfloor \bar{\zeta}_{0}\right) \\
& \left.\left.\left.\quad=\left(\frac{1}{n+2} d \gamma+\frac{1}{2(n+1)} \alpha_{\zeta_{0}} \theta\right) \wedge\left(i d \gamma \wedge(T\rfloor \zeta_{0}\right)+d(T\rfloor \zeta_{0}\right)\right) \wedge(T\rfloor \bar{\zeta}_{0}\right) \\
& \left.\left.\left.\left.\quad=\frac{1}{n+2} d \gamma \wedge d(T\rfloor \varsigma_{0}\right) \wedge(T\rfloor \bar{\zeta}_{0}\right)+\frac{i}{2(n+1)} \alpha_{\varsigma_{0}} \theta \wedge d \gamma \wedge(T\rfloor \zeta_{0}\right) \wedge(T\rfloor \bar{\zeta}_{0}\right)
\end{aligned}
$$

or

$$
\left.\left.\left.\left.i \alpha_{\varsigma_{0}} d \gamma \wedge \theta \wedge(T\rfloor \varsigma_{0}\right) \wedge(T\rfloor \bar{\zeta}_{0}\right)=d \gamma \wedge d(T\rfloor \varsigma_{0}\right) \wedge(T\rfloor \bar{\zeta}_{0}\right)
$$

But this is just the wedge product of equation (5.20) with $d \gamma$, which proves the result.

It follows from the above proof and from the arguments in [4] that (5.21) satisfies (3.5) iff $u$ satisfies (5.18) along $M$, and satisfies (3.6) iff $u$ satisfies (5.18) to second order. This is the sense in which the two normalizations of $\sigma$ correspond to the first and second formal power series solutions to the complex Monge-Ampère equation. 
6. Curvature of the Fefferman metric. In this section we use the characterizations of the Fefferman metric introduced in $\S \S 3$ and 5 to compute curvature invariants of the metric in terms of pseudohermitian invariants of $M$.

We begin by comparing the Laplace-Beltrami operator $\square$ of $g$ to the sublaplacian $\Delta_{b}$. Since $g$ is invariant under the natural $S^{1}$ action on $C, \square$ pushes forward to an operator $\pi_{*} \square$ on $M$ in the following way. For $u \in C^{\infty}(M)$, we set $\left(\pi_{*} \square\right) u=$ $\square\left(\pi^{*} u\right)$, which descends to a function on $M$ since $\pi^{*} u$ is constant on fibers of $C$ and $\square$ is $S^{1}$-invariant. Then we have the following result:

(6.1) Proposition. $\pi_{*} \square=2 \Delta_{b}$.

ProOF. Recall that $\square$ is defined on functions by

$$
\int_{C}(\square u) \bar{v} \mu=\int_{C} g^{*}(d u, d \bar{v}) \mu
$$

for all $v \in C_{c}^{\infty}(C)$, where $\mu$ denotes the metric volume form on $C$. Let $\varsigma_{0}$ be a volume-normalized $(n+1,0)$-form on $M$, so that $\zeta=e^{i \gamma} \zeta_{0}$ is the canonical $(n+1)$-form on $C$, and $\psi=\theta \wedge d \theta^{n-1}$. We claim that $\mu=c_{n} d \gamma \wedge \psi$ for some universal constant $c_{n}$. If $\left\{\theta^{\alpha}\right\}$ is an admissible coframe in which $h_{\alpha \bar{\beta}}=\delta_{\alpha \bar{\beta}}$, then $\psi=i^{n^{2}} n ! \theta \wedge \theta^{1} \wedge \cdots \wedge \theta^{n} \wedge \theta^{\overline{1}} \wedge \cdots \wedge \theta^{\bar{n}}$.

From formula (5.2) for $g$ it follows that $\left\{\operatorname{Re} \theta^{\alpha}, \operatorname{Im} \theta^{\alpha}, 2^{-1 / 2}(\theta+\sigma), 2^{-1 / 2}(\theta-\sigma)\right\}$ is an orthonormal coframe for $g$, and so for some universal constants $b_{n}, c_{n}$

$$
\mu=b_{n} \sigma \wedge \theta \wedge \theta^{1} \wedge \cdots \wedge \theta^{n} \wedge \theta^{\overline{1}} \wedge \cdots \wedge \theta^{\bar{n}}=c_{n} d \gamma \wedge \psi
$$

Let $\left\{N, W_{\alpha}, W_{\bar{\alpha}}, \Sigma\right\}$ be the dual frame to $\left\{\theta, \theta^{\alpha}, \theta^{\bar{\alpha}}, \sigma\right\}$ on $C$. Note that $\Sigma=$ $S /(n+2)$, and $N$ is the unique lift of $T$ such that $L_{\theta}(N, N)=0$. The dual metric $g^{*}$ to $g$ is $g^{*}=L_{\theta}^{*}+2 N \cdot \Sigma$. Thus for $u \in C^{\infty}(M), v \in C_{c}^{\infty}(M), g^{*}\left(\pi^{*} d u, \pi^{*} d v\right)=$ $L_{\theta}^{*}\left(d_{b} u, d_{b} v\right)$, and so

$$
\begin{aligned}
& \int_{M}\left(\left(\pi_{*} \square\right) u\right) \bar{v} \psi=\frac{1}{2 \pi} \int_{C}\left(\square\left(\pi^{*} u\right)\right) \pi^{*} \bar{v} d \gamma \wedge \psi \\
& \quad=\frac{1}{2 \pi} \int_{C} g^{*}\left(\pi^{*} d u, \pi^{*} d \bar{v}\right) d \gamma \wedge \psi=\frac{1}{2 \pi} \int_{C} L_{\theta}^{*}\left(d_{b} u, d_{b} \bar{v}\right) d \gamma \wedge \psi \\
& \quad=\int_{M} L_{\theta}^{*}\left(d_{b} u, d_{b} \bar{v}\right) \psi=2 \int_{M}\left(\Delta_{b} u\right) \bar{v} \psi .
\end{aligned}
$$

We turn now to the scalar curvature. Let $K$ denote the scalar curvature of $g$ on $C$, and $R$ the Webster scalar curvature of $\theta$ on $M$. Note that by the $S^{1}$ invariance of $g, K$ is constant on the fibers of $C$, and thus descends to a function $\pi_{*} K$ on $M$.

(6.2) THEOREM. $\pi_{*} K=2(2 n+1) R /(n+1)$.

PROOF. This will follow from Theorem 6.6, but we present here an easier proof using invariant theory.

Suppose $\tilde{\theta}=e^{2 f} \theta$ is a new pseudohermitian structure on $M$. A standard calculation (cf., for example, [1]) shows that, under the conformal change of metric $\tilde{g}=e^{2 f} g, K$ transforms by

$$
\tilde{K}=e^{-2 f}\left(K+2(2 n+1) \square\left(\pi^{*} f\right)-2 n(2 n+1) g^{*}\left(\pi^{*} d f, \pi^{*} d f\right)\right)
$$


(recall that $\operatorname{dim} C=2 n+2$ ). On the other hand, Proposition 5.15 shows that

$$
\tilde{R}=e^{-2 f}\left(R+2(n+1) \Delta_{b} f-n(n+1) L_{\theta}^{*}\left(d_{b} f, d_{b} f\right)\right) .
$$

Consider the function $D_{\theta}=\pi_{*} K-2(2 n+1) R /(n+1)$, defined on $M$ for any pseudohermitian structure $\theta$. Then Proposition 6.1 and the above calculations show that if $\tilde{\theta}=e^{2 f} \theta, D_{\tilde{\theta}}=e^{-2 f} D_{\theta}$. In other words, the assignment $\theta \mapsto D_{\theta}$ determines a scalar invariant of the CR structure of $M$ (of weight 2). We will show that $D_{\theta}$ is identically zero, which proves the theorem.

Using the Moser normal form [3] for $M$ at a point $p \in M$, we can embed $M$ locally in $\mathbf{C}^{n+1}$ with coordinates $\left(w=u+i v, z^{1}, \ldots, z^{n}\right)$ such that $M$ is approximated to arbitrarily high order at $p$ by the power series

$$
0=\rho(w, z)=-v+|z|^{2}+\sum_{|A|,|B| \geq 2} \sum_{m} a_{A B m} z^{A} \bar{z}^{B} u^{m}
$$

where $A, B$ are multi-indices. The coefficients $a_{A B m}$ are determined up to a finitedimensional symmetry group by additional normalizations. Since $D_{\theta}(0)$ depends only on the value of $\theta$ at 0 , we can calculate it by choosing any normal form with $\theta=i(\bar{\partial} \rho-\partial \rho) / 2$ at 0 .

We note first that the vector field $T$ associated with $\theta$ is determined by the relations

$$
T\rfloor \partial \bar{\partial} \rho=T\rfloor d \rho=0, \quad T\rfloor \theta=1 .
$$

Since $\partial \bar{\partial} \rho=\sum_{j} d z^{j} \wedge d z^{\bar{j}}$ and $d \rho=-d v$ at 0 , one can verify that the Taylor coefficients of the components of $T$ in terms of $\left\{\partial / \partial u, \partial / \partial v, \partial / \partial z^{j}, \partial / \partial z^{\bar{j}}\right\}$ are given by polynomials in the $a_{A B m}$. We can take $\left\{\theta^{\alpha}=d z^{\alpha}-\left(T z^{\alpha}\right) \theta\right\}$ as an admissible coframe. With respect to this coframe, the Taylor coefficients of the connection forms $\omega_{\alpha}{ }^{\beta}$ and curvature forms $\Pi_{\alpha}{ }^{\beta}$ at 0 are again given by polynomials in the $a_{A B m}$. It then follows from the discussion in $\S \S 4$ and 5 that the values of $R$ and $K$, and thus also of $D_{\theta}$, at 0 are given by a universal polynomial in the coefficients $a_{A B m}$.

Now the coordinate change $\tilde{w}=e^{2 \lambda} w, \tilde{z}=e^{\lambda} z$, with $\lambda$ a real constant, yields the normal form

$$
\begin{aligned}
\tilde{\rho} & =-\tilde{v}+|\tilde{z}|^{2}+\sum_{|A|,|B| \geq 2} \sum_{m} \tilde{a}_{A B m} \tilde{z}^{A} \overline{\tilde{z}}^{B} \tilde{u}^{m} \\
& =e^{2 \lambda}\left(-v+|z|^{2}+\sum_{|A|,|B| \geq 2} \sum_{m} e^{(|A|+|B|+2 m-2) \lambda} \tilde{a}_{A B m} z^{A} \bar{z}^{B} u^{m}\right),
\end{aligned}
$$

which shows we can take $\tilde{a}_{A B m}=e^{-(|A|+|B|+2 m-2) \lambda} a_{A B m}$, so that $\tilde{\rho}=e^{2 \lambda} \rho$ and $\tilde{\theta}=e^{2 \lambda} \theta$.

From the preceding discussion,

$$
D_{\tilde{\theta}}(0)=P\left(\tilde{a}_{A B m}\right)=P\left(e^{-(|A|+|B|+2 m-2) \lambda} a_{A B m}\right)=e^{-2 \lambda} P\left(a_{A B m}\right) .
$$

Since $a_{A B m}=0$ for $|A|+|B|<4$, this means that $P$ can involve only the lowestorder terms $a_{A B m}$, with $|A|=|B|=2$ and $m=0$, and $P$ must be linear in these terms.

Now the symmetry group $H$ of the normal form contains the unitary group $U(n)$ acting on the $z$ variables, and under this representation the coefficients $a_{A B 0}$ with 
$|A|=|B|=2$ transform as the components of a tensor of bidegree $(2,2)$ on $\mathbf{C}^{n}$. Thus by classical invariant theory, the only nontrivial linear scalar invariant under $U(n)$ is the complete contraction

$$
\sum h^{\alpha_{1} \bar{\beta}_{1}} h^{\alpha_{2} \bar{\beta}_{2}} a_{\left(\alpha_{1} \alpha_{2}\right)\left(\beta_{1} \beta_{2}\right) 0}
$$

But by the normalization conditions defining the normal form (cf. [3]), this trace is already zero. Thus $P$ is the zero polynomial.

It is remarkable that, although the Fefferman metric depends on two derivatives of the CR structure (as represented, say, by a coframe $\left\{\theta, \theta^{\alpha}\right\}$ ) and therefore its curvature depends on four derivatives, its scalar curvature turns out, through nonobvious cancellations, to involve only two derivatives.

Next we compute the connection 1-forms of the Fefferman metric. We will work with the frame for the complexified tangent bundle CTC

$$
\left(X_{0}, \ldots, X_{2 n+1}\right)=\left(N, Y_{1}, \ldots, Y_{n}, Y_{\overline{1}}, \ldots, Y_{\bar{n}}, \Sigma\right)
$$

dual to the coframe

$$
\left(\xi^{0}, \ldots, \xi^{2 n+1}\right)=\left(\theta, \theta^{1}, \ldots, \theta^{n}, \theta^{\overline{1}}, \ldots, \theta^{\bar{n}}, \sigma\right) .
$$

Let us write $Z_{\alpha}=\pi_{*} Y_{\alpha} \in T_{1,0}$.

With respect to this frame, the metric is represented by the matrix:

$$
\left(g_{j k}\right)=\left[\begin{array}{cccc}
0 & 0 & 0 & 1 \\
0 & 0 & \frac{1}{2} h_{\alpha \bar{\beta}} & 0 \\
0 & \frac{1}{2} h_{\bar{\alpha} \beta} & 0 & 0 \\
1 & 0 & 0 & 0
\end{array}\right] .
$$

(Here and in the remainder of this section, we let lower case Greek indices run from 1 to $n$, lower case Roman indices from 0 to $2 n+1$, and write $\bar{\alpha}=\alpha+n$.)

The Levi-Civita connection $\left(\phi_{j}{ }^{k}\right)$ of the metric is determined by the structure equations

$$
\begin{gathered}
d \xi^{j}=\xi^{k} \wedge \phi_{k}{ }^{j} \\
\phi_{j}{ }^{l} g_{l k}+\phi_{k}{ }^{l} g_{l j}=d g_{j k} .
\end{gathered}
$$

(6.5) Proposition. The Levi-Civita connection of $g$ is given by

$$
\left(\phi_{j}{ }^{k}\right)=\left[\begin{array}{cccc}
0 & i \sigma^{\beta} & -i \sigma^{\bar{\beta}} & 0 \\
\frac{i}{2} \theta_{\alpha} & \phi_{\alpha}{ }^{\beta} & 0 & \frac{i}{2} \sigma_{\alpha} \\
-\frac{i}{2} \theta_{\bar{\alpha}} & 0 & \phi_{\bar{\alpha}}{ }^{\bar{\beta}} & -\frac{i}{2} \sigma_{\bar{\alpha}} \\
0 & i \theta^{\beta} & -i \theta^{\bar{\beta}} & 0
\end{array}\right]
$$

where

$$
\begin{gathered}
\phi_{\alpha}{ }^{\beta}=\overline{\phi_{\bar{\alpha}}^{\bar{\beta}}}=\omega_{\alpha}^{\beta}+i K_{\alpha}^{\beta} \theta+i \delta_{\alpha}^{\beta} \sigma, \quad \sigma_{\alpha}=i A_{\alpha \gamma} \theta^{\gamma}+K_{\alpha \bar{\gamma}} \theta^{\bar{\gamma}}+C_{\alpha} \theta \\
K_{\alpha \bar{\beta}}=\frac{1}{n+2}\left(R_{\alpha \bar{\beta}}-\frac{1}{2(n+1)} R h_{\alpha \bar{\beta}}\right), \quad C_{\alpha}=\frac{2}{n+2}\left(W_{\alpha}+\frac{i}{2(n+1)} Z_{\alpha} R\right), \\
W_{\alpha}=W_{\beta_{\alpha}{ }_{\alpha}}=W^{\bar{\beta}} \bar{\beta}_{\alpha} .
\end{gathered}
$$


Here $A_{\alpha \gamma}, R_{\alpha \bar{\beta}}, R$, and $W_{\alpha}{ }^{\beta}{ }_{\gamma}$ are the pseudohermitian invariants defined in (4.4), (4.7) and (4.8).

PROOF. The fact that $\left(\phi_{j}{ }^{k}\right)$ satisfies (6.4) is a straightforward matrix calculation, using (4.2) and the fact that $K_{\alpha \bar{\beta}}=\bar{K}_{\beta \bar{\alpha}}=K_{\bar{\beta} \alpha}$.

To verify $(6.3)$, we compute

$$
\begin{aligned}
& \xi^{j} \wedge \phi_{j}{ }^{0}=\theta^{\alpha} \wedge \frac{i}{2} \theta_{\alpha}-\theta^{\bar{\alpha}} \wedge \frac{i}{2} \theta_{\bar{\alpha}}=i h_{\alpha \bar{\beta}} \theta^{\alpha} \wedge \theta^{\bar{\beta}}=d \theta, \\
\xi^{j} \wedge \phi_{j}{ }^{\beta}= & \theta \wedge i \sigma^{\beta}+\theta^{\alpha} \wedge \phi_{\alpha}{ }^{\beta}+\theta^{\bar{\alpha}} \wedge \phi_{\bar{\alpha}}{ }^{\beta}+\sigma \wedge i \theta^{\beta} \\
= & \theta \wedge i\left(-i A^{\beta} \bar{\gamma} \theta^{\bar{\gamma}}+K^{\beta}{ }_{\gamma} \theta^{\gamma}+C^{\beta} \theta\right) \\
& +\theta^{\alpha} \wedge\left(\omega_{\alpha}{ }^{\beta}+i K_{\alpha}{ }^{\beta} \theta+i \delta_{\alpha}^{\beta} \sigma\right)+\sigma \wedge i \theta^{\beta} \\
= & \theta^{\alpha} \wedge \omega_{\alpha}{ }^{\beta}+\theta \wedge \tau^{\beta}=d \theta^{\beta}, \\
\xi^{j} \wedge \phi_{j}{ }^{2 n+1}= & \theta^{\alpha} \wedge \frac{i}{2} \sigma_{\alpha}-\theta^{\bar{\alpha}} \wedge \frac{i}{2} \sigma_{\bar{\alpha}} \\
= & -\frac{1}{2} A_{\alpha \gamma} \theta^{\alpha} \wedge \theta^{\gamma}+\frac{i}{2} K_{\alpha \bar{\gamma}} \theta^{\alpha} \wedge \theta^{\bar{\gamma}}+\frac{i}{2} C_{\alpha} \theta^{\alpha} \wedge \theta-\frac{1}{2} A_{\overline{\alpha \gamma}} \theta^{\bar{\alpha}} \wedge \theta^{\bar{\gamma}} \\
& -\frac{i}{2} K_{\bar{\alpha} \gamma} \theta^{\bar{\alpha}} \wedge \theta^{\gamma}-\frac{i}{2} C_{\bar{\alpha}} \theta^{\bar{\alpha}} \wedge \theta \\
= & i K_{\alpha \bar{\gamma}} \theta^{\alpha} \wedge \theta^{\bar{\gamma}}+\frac{i}{2} C_{\alpha} \theta^{\alpha} \wedge \theta-\frac{i}{2} C_{\bar{\alpha}} \theta^{\bar{\alpha}} \wedge \theta .
\end{aligned}
$$

On the other hand, from (5.3) and (4.7),

$$
\begin{aligned}
d \sigma= & \frac{1}{n+2}\left(i d \omega_{\alpha}{ }^{\alpha}-\frac{1}{2(n+1)} d R \wedge \theta-\frac{1}{2(n+1)} R d \theta\right) \\
= & \frac{1}{n+2}\left(i R_{\alpha \bar{\gamma}} \theta^{\alpha} \wedge \theta^{\bar{\gamma}}+i W_{\alpha} \theta^{\alpha} \wedge \theta-i W_{\bar{\alpha}} \theta^{\bar{\alpha}} \wedge \theta-\frac{1}{2(n+1)} Z_{\alpha} R \theta^{\alpha} \wedge \theta\right. \\
& \left.\quad-\frac{1}{2(n+1)} Z_{\bar{\alpha}} R \theta^{\bar{\alpha}} \wedge \theta-\frac{1}{2(n+1)} i R h_{\alpha \bar{\gamma}} \theta^{\alpha} \wedge \theta^{\bar{\gamma}}\right) \\
= & i K_{\alpha \bar{\gamma}} \theta^{\alpha} \wedge \theta^{\bar{\gamma}}+\frac{i}{2} C_{\alpha} \theta^{\alpha} \wedge \theta-\frac{i}{2} C_{\bar{\alpha}} \theta^{\bar{\alpha}} \wedge \theta .
\end{aligned}
$$

So $d \xi^{2 n+1}=d \sigma=\xi^{j} \wedge \phi_{j}{ }^{2 n+1}$, and $\left(\phi_{j}{ }^{k}\right)$ satisfies (6.3).

Finally, we state the following formula for the Ricci tensor of the Fefferman metric. The proof is a very long and laborious calculation based on Proposition 6.5 , and is omitted here.

(6.6) THEOREM. The Ricci tensor of the Fefferman metric is

$$
\rho=\frac{1}{n+1} R g+n \theta^{\gamma} \cdot\left(\sigma_{\gamma}+C_{\gamma} \theta\right)+n \theta^{\bar{\gamma}} \cdot\left(\sigma_{\bar{\gamma}}+C_{\bar{\gamma}} \theta\right)+2 n \sigma^{2}+\lambda \theta^{2}
$$

where

$$
\lambda=2 K_{\alpha \bar{\beta}} K^{\alpha \bar{\beta}}-2 A_{\alpha \beta} A^{\alpha \beta}+\frac{4}{n+2} \operatorname{Im} A_{\alpha \beta}{ }^{\alpha \beta}-\frac{1}{(n+1)(n+2)} \Delta_{b} R .
$$


This result yields another proof of Theorem 6.2, simply by contracting $\rho_{j k}$ with $g_{j k}$.

This expression for the Ricci tensor shows, for example, that the Fefferman metric is never Einstein. It is hoped that the simple relationship of the Ricci tensor to the pseudohermitian invariants of $M$ will contribute to a better understanding of the geometry of the Fefferman metric.

\section{BIBLIOGRAPHY}

1. T. Aubin, Métriques Riemanniennes et Courbure, J. Differential Geom. 4 (1970), 383-424.

2. D. Burns, K. Diederich and S. Shnider, Distinguished curves in pseudoconvex boundaries, Duke Math. J. 44 (1977), 407-431.

3. S. S. Chern and J. K. Moser, Real hypersurfaces in complex manifolds, Acta Math. 133 (1974), 219-271.

4. F. Farris, An intrinsic construction of Fefferman's $C R$ metric, Pacific J. Math. (to appear).

5. C. Fefferman, Monge-Ampère equations, the Bergman kernel, and geometry of pseudoconvex domains, Ann. of Math. (2) 103 (1976), 395-416; correction, 104 (1976), 393-394.

6. G. B. Folland and E. M. Stein, Estimates for the $\bar{\partial}_{b}$-complex and analysis on the Heisenberg group, Comm. Pure Appl. Math. 27 (1974), 429-522.

6a.C. R. Graham, On Sparling's characterization of Fefferman metrics, preprint, 1986.

7. A. Greenleaf, The first eigenvalue of a sub-Laplacian on a pseudohermitian manifold, Comm. Partial Differential Equations 10 (1985), 191-217.

7a.H. Jacobowitz, The canonical bundle and realizable CR hypersurfaces, preprint, 1985.

8. D. Jerison and J. Lee, A subelliptic, nonlinear eigenvalue problem and scalar curvature on $C R$ manifolds, Contemp. Math. 27 (1984), 57-63.

9. , The Yamabe problem on CR manifolds, preprint, 1985.

10. J. J. Kohn, Boundaries of complex manifolds, Proc. Conf. on Complex Analysis Minneapolis, 1964, Springer-Verlag, New York, 1965, pp. 81-94.

11. C. Stanton, Intrinsic connections for Levi metrics, prerint, 1983.

12. S. M. Webster, Pseudohermitian structures on a real hypersurface, J. Differential Geom. 13 (1978), 25-41.

Department of Mathematics, Harvard University, Cambridge, MassachuSETTS 02138 